\title{
Potassium Channel Kv1.3 Is Highly Expressed by Microglia in Human Alzheimer's Disease
}

\author{
Srikant Rangaraju ${ }^{\mathrm{a}, *}$, Marla Gearing ${ }^{\mathrm{a}}$, Lee-Way Jin ${ }^{\mathrm{b}}$ and Allan Levey ${ }^{\mathrm{a}}$ \\ ${ }^{a}$ Department of Neurology, Emory University, Atlanta, GA, USA \\ ${ }^{\mathrm{b}}$ Department of Pathology and Laboratory Medicine, Alzheimer's Disease Center, University of California Davis, \\ CA, USA
}

Accepted 23 September 2014

\begin{abstract}
Recent genetic studies suggest a central role for innate immunity in Alzheimer's disease (AD) pathogenesis, wherein microglia orchestrate neuroinflammation. Kv1.3, a voltage-gated potassium channel of therapeutic relevance in autoimmunity, is upregulated by activated microglia and mediates amyloid-mediated microglial priming and reactive oxygen species production in vitro. We hypothesized that Kv1.3 channel expression is increased in human AD brain tissue. In a blinded postmortem immunohistochemical semi-quantitative analysis performed on ten AD patients and ten non-disease controls, we observed a significantly higher Kv1.3 staining intensity $(p=0.03)$ and Kv1.3-positive cell density $(p=0.03)$ in the frontal cortex of AD brains, compared to controls. This paralleled an increased number of Iba1-positive microglia in AD brains. Kv1.3-positive cells had microglial morphology and were associated with amyloid- $\beta$ plaques. In immunofluorescence studies, Kv1.3 channels co-localized primarily with Iba1 but not with astrocyte marker GFAP, confirming that elevated Kv1.3 expression is limited to microglia. Higher Kv1.3 expression in AD brains was also confirmed by western blot analysis. Our findings support that Kv1.3 channels are biologically relevant and microglia-specific targets in human AD.
\end{abstract}

Keywords: Alzheimer's disease, Kv1.3, microglia, neuroinflammation, potassium channel

\section{INTRODUCTION}

Microglia represent the innate immunity of the central nervous system (CNS) and like macrophages, perform pro-inflammatory as well as regulatory functions [1]. Microglia induced by $\mathrm{T}$ cell cytokines tumor necrosis factor $\alpha$ and interferon $\gamma$ are proinflammatory while some microglia have regulatory and anti-inflammatory functions [2]. In Alzheimer's disease (AD), microglia surround amyloid plaques and contribute to amyloid turnover. It is suggested that early microglial activation in AD has a neuroprotective effect by promoting clearance of amyloid- $\beta$

\footnotetext{
*Correspondence to: Srikant Rangaraju, MD, 615 Michael Street, Whitehead Research Building, Room 525, Atlanta, GA 30322, USA. Tel.: +1 404727 7220; Fax: +1 404727 3999; E-mail: srangar@emory.edu.
}

(A $\beta)$, however, in the later stages pro-inflammatory cytokines produced by activated microglia have detrimental effects that promote neurodegeneration [3]. The central role of microglia in AD pathogenesis is further supported by genetic studies that identified an AD susceptibility locus in CD33, and an increased risk of AD associated with allelic variants in Triggering Receptor Expressed on Myeloid cells-2 (TREM2), both microglial proteins [4-6].

Microglia are the key inflammatory cells in AD that mediate neuroinflammation, and voltage-gated Kv1.3 potassium channels are key regulators of microglial function. Resting microglia express Kv1.5 channels and upon activation and proliferation, upregulate Kv1.3 and down-regulate Kv1.5 channels. Kv1.3 channels also migrate to the cell surface while Kv1.5 channels are internalized making Kv1.3 channels not only functionally relevant but also most susceptible 
to blockade by highly selective channel blockers [7, 8]. Peptide blockers of Kv1.3 channels (margatoxin and the highly-specific sea anemone toxin analogs ShK-186, ShK-170, ShK-192) and non-peptide blockers (PAP-1) have been developed as experimental therapeutics in multiple sclerosis and other autoimmune disorders, where they act primarily by blocking Kv1.3 channels on autoimmune effector-memory $\mathrm{T}$ cells [9]. A better understanding of microglial potassium channel regulation and expression patterns in neurodegenerative states could also yield targets for drug development using potassium channel blockers.

In animal models of $\mathrm{AD}$, microglial priming by $\mathrm{A} \beta$ depends on Kv1.3 channels [7, 8]. Therapeutic targeting of Kv1.3 channels in AD with selective blockers could be justified if Kv1.3 channel expression is increased in a cell-specific manner in human tissue. In this study, we have investigated Kv1.3 channel expression in human AD brain tissue, as compared to non-AD controls. Furthermore, we characterized patterns of Kv1.3 channel expression and determined the cellular localization of Kv1.3 channels in frontal cortex of brains from AD patients. Our results provide evidence that supports the possibility that Kv1.3 channels represent therapeutic targets in AD.

\section{MATERIALS AND METHODS}

\section{Brain tissue}

Ten AD brains and 10 age-matched non-disease control cryopreserved brain tissues were randomly selected from a neuropathological repository from the Emory Alzheimer Disease Research Center Neuropathology Core, Atlanta. $12 \mu \mathrm{m}$ sections from paraffin-embedded frontal cortical brain tissue were prepared. Mean age was $71.5 \pm 3.6$ years and $71.5 \pm 3.8$ years in cases and controls respectively. Other baseline characteristics are described in the supplementary material (Supplementary Table 1). The samples were coded for blinded immunohistochemical analysis.

\section{Antibodies}

Rabbit anti-human Kv1.3 polyclonal IgG (\#APC101) was obtained from Alomone Laboratories, Jerusalem. Optimization for immunohistochemistry (IHC) on paraffin embedded tissue was performed on control brain tissue as well as mouse spleen tissue (positive control) and dilution of $1: 100$ was selected. Goat anti-Iba1 polyclonal Ab (Abcam \#ab5076) was used at 1:200 dilution. Appropriate goat anti-rabbit (Sigma-Aldrich, 1:1000) and horse anti-goat IgG (Sigma, $1: 1000$ ) were used as secondary antibodies. Anti-GFAP mAb (Millipore MB360) was used to label astrocytes and Anti-A $\beta$ monoclonal 4G8 (A $\beta$ 17-24) antibody (Covance SIG 39245) was used to identify amyloid plaques.

\section{Immunohistochemistry on paraffin sections}

Paraffin-embedded sections were deparaffinized through an alcohol gradient, heated in a microwave in $10 \mathrm{mM}$ citric acid (pH 6.0) for $15 \mathrm{~min}$, blocked with $0.3 \%$ hydrogen peroxide for $30 \mathrm{~min}$ and then blocked with $4 \%$ normal goat serum $/ 0.4 \%$ Triton X100/Tris-buffered saline (TBS) for $1 \mathrm{~h}$, followed by overnight incubation with primary anti-Kv1.3 antibody or anti-Ibal antibody. Sections were rinsed with TBS and incubated with appropriate biotin-conjugated secondary antibody followed by Vectastain Elite ABC and diaminobenzidine (DAB) as per manufacturer's recommendations. To exclude nonspecific staining unrelated to polyclonal and monoclonal antibodies, immunostaining was performed with omission of the antibodies but with all other procedures unchanged in some experiments. Slides were lastly counterstained with hematoxylin. Specificity of anti-Kv1.3 antibody was determined in two AD samples by pre-adsorption with Kv1.3 peptide ( $8 \mu \mathrm{g} / \mathrm{ml}$, Alomone laboratories) followed by further immunostaining with primary and secondary antibodies as described above.

For double antigen immunohistochemistry for Kv1.3 and $A \beta$, slides were first immunostained with anti-Kv1.3 Ab, secondary Ab and developed with Vectastain Elite $\mathrm{ABC}$ and $\mathrm{DAB}$ chromogen. This was followed by antigen retrieval with $98 \%$ formic acid and incubation with anti-A $\beta$ antibody (4G8, $1: 2000)$ for $1 \mathrm{~h}$. Secondary $\mathrm{Ab}$ incubation was followed by Vectastain Elite ABC and SG-Blue chromogen per manufacturer's protocol. Light microscopy was performed with an Olympus light microscope (Olympus, Center Valley, PA).

\section{Pathologic grading}

All AD and control specimens that were immunostained for Kv1.3 and Iba1 were independently evaluated by two investigators (SR and AL). Staining intensity of positive cells at 20x magnification, based on intensity of DAB labelling, was graded as follows: 0 - no staining, 1 - mild, 2 - moderate, 3 - strong. Examples of each grade were provided to each observer 
for reference. Staining density, defined as number of positive cells per 20x power field, was graded as follows: 0 - no positive cells/field, $1-1-10 /$ field, $2-$ 11-50/field, $3-\geq 50 /$ field. Each observer rated 3 cortical fields and 3 white matter fields per slide for staining intensity and density and the mean score was used as a final grade. Inter-observer agreement for staining intensity as well as staining density was calculated. Mean staining intensity and staining density was compared between AD cases and controls using Student's $t$-test ( $p \leq 0.05$ was considered significant for a twotailed analysis).

\section{Immunofluorescence microscopy and co-localization analysis}

Slides from $3 \mathrm{AD}$ cases and 3 controls were incubated with rabbit anti-Kv1.3 $(1: 100)$ and goat anti-Iba1 $(1: 200)$ or rabbit anti-Kv1.3 and mouse anti-GFAP mAb (1:500) antibodies. Phycoerythrinconjugated Donkey anti-rabbit IgG $(1: 1000)$, Fluorescein Isothiocyanate (FITC)-conjugated donkey anti-goat, and FITC-conjugated goat anti-mouse IgG $(1: 1000)$ were used as secondary antibodies. Three AD cases were also immunostained with anti-neuronspecific $\beta$ tubulin III (Tuj1, Promega G712A, $1: 100$ ) and anti-Kv1.3 antibodies. Pre-incubation of tissue with $10 \mathrm{mM}$ cupric sulfate for $30 \mathrm{~min}$ was performed to reduce autofluorescence due to lipofuscin. Single antibody controls for each channel were used to optimize settings for image acquisition. Non-specific staining was evaluated by omission of primary antibodies but with all other steps. Images were acquired at $20 \mathrm{x}$ and 40x magnification on an Olympus microscope (Microscope: Olympus BX51 and camera: Olympus DP70). Three representative images in each group at 20x magnification were picked for co-localization analysis using ImageJ software [10]. Degree or percentage co-localization (Supplementary Fig. 1) was defined as the percentage of pixels positive on both channels, expressed over a denominator of all pixels that were positive on either channel. Thresholds for positivity were defined based on control slides where primary antibody was omitted. Average percentage co-localization $(n=3)$ in AD cases and controls were compared using Student's $t$-test ( $p \leq 0.05$ was considered significant for a two-tailed analysis).

For fluorescence microscopic visualization of amyloid plaques using Thioflavin-S, slides immunostained for Kv1.3 or Iba1 were incubated with freshly prepared aqueous $1 \%$ filtered Thioflavin-S for 10 min (SigmaAldrich, T1892) at room temperature, washed thrice in $80 \%$ ethanol and finally with $95 \%$ ethanol, and mounted in a aqueous mounting medium [11]. Iba1 or Kv1.3 positive regions were first visualized by light microscopy and amyloid positivity was subsequently visualized with a FITC filter. For co-localization experiments using immunofluorescence microscopy, appropriate FITC-conjugated antibodies for anti-Iba1 primary or anti-GFAP primary and PE-conjugated antirabbit secondary antibodies were used. After nuclear staining with $4^{\prime}, 6^{\prime}$-diamidino-2-phenylindole (DAPI) for $10 \mathrm{~min}$, sections were cover-slipped with glycerol, and fluorescent images were obtained with a fluorescence microscope (Microscope: Olympus BX51 and camera: Olympus DP70) using FITC, PE, and DAPI filters and images were processed using ZEISS LSM 5 Series Image Browser.

\section{Tissue preparation and western blot analysis}

Membrane-enriched protein extracts were prepared as previously described [12] from frozen frontal cortical tissue of $4 \mathrm{AD}$ and 5 control brains that had been included in immunohistochemistry studies. Briefly, approximately $200 \mathrm{mg}$ of brain tissue was homogenized in lysis buffer $(10 \mathrm{mM}$ Tris base/hydrochloride, $10 \mathrm{mM}$ EDTA, pH 7.4) with $1 \mathrm{X}$ protease inhibitor cocktail (\#13006200; Roche, San Francisco, CA) while on ice followed by agitation with metallic beads and sonication $(4 \times 5$-s pulses with $15 \mathrm{~s}$ breaks to avoid overheating). Suspensions were then centrifuged at $22,000 \mathrm{~g}$ for $15 \mathrm{~min}$ and the pellet was solubilized in $8 \mathrm{M}$ Urea on ice for $1 \mathrm{~h}$ followed by centrifugation. The supernatant was collected and protein estimation (Pierce ${ }^{\mathrm{TM}}$ BCA Protein Assay Kit, \#23227) was performed. Sodium dodecyl sulfate (SDS) sample buffer with [beta]-mercaptoethanol (MP Biomedicals, Solon, $\mathrm{OH}$ ) was added to $50 \mu \mathrm{g}$ of protein from each sample and loaded onto $10 \%$ SDS denaturing gels. Western blots were performed per standard protocols. After overnight protein transfer, PVDF membrane was blocked with $5 \%$ fat-free milk for $1 \mathrm{~h}$ followed by incubation with primary antibodies. The total protein samples were incubated with anti-Kv1.3 rabbit polyclonal antibody (APC 101, Alomone labs, 1:1000) and anti- $\alpha$-tubulin (Cell Signaling, \#3873, $1: 1000$ ) for $24 \mathrm{~h}$. Membrane fraction samples were probed with anti-Kv1.3 and anti-Na/K ATPase $\alpha 1$ subunit mouse monoclonal antibody (Millipore \#05-369, 1 : 1000) for $2 \mathrm{~h}$. After 3 washes, fluorescent secondary antibodies (anti-mouse IgG IRDye ${ }^{\circledR} 800$ conjugate, Rockland, $1: 20,000$ and anti-rabbit IgG Alexa Fluor ${ }^{\circledR} 680$ conjugate, Invitrogen) were added for $1 \mathrm{~h}$. An Odyssey 

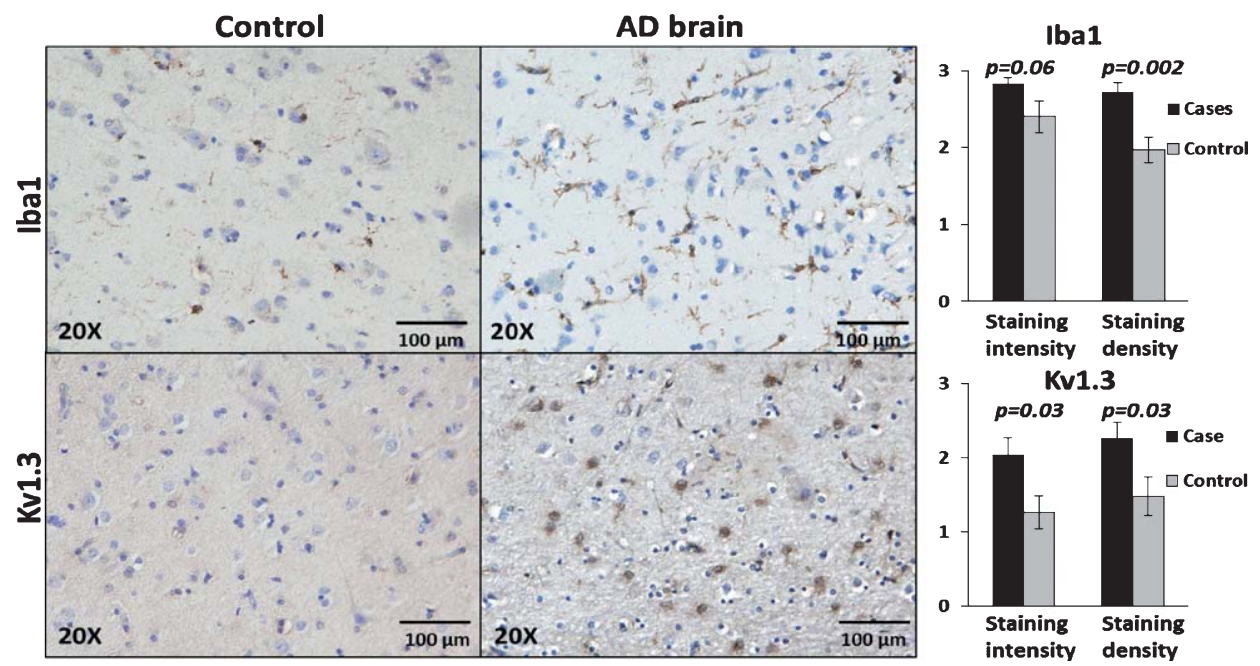

Fig. 1. Iba1 and Kv1.3 expression is increased in AD brains as compared to control brains. Left: Representative 20x images demonstrating low Iba1 and minimal Kv1.3 staining in a control brain (top panel) compared to strong Iba1 positivity and robust Kv1.3 positivity in an AD brain (bottom panel). Right: Statistical comparison of Iba1 (top panel) and Kv1.3 (bottom panel) staining intensity and staining density in cortical regions. $P$-values for significant differences between cases and controls are indicated.

Scanner (LI-COR, Lincoln, NE) was used to visualize proteins labeled and densitometric analysis of gel bands was performed using ImageJ as previously described [12]. Kv1.3 protein expression was normalized to $\mathrm{Na} / \mathrm{K}$ ATPase $\alpha 1$ subunit (for membrane fractions) and both absolute and mean densitometric units were compared across both groups after three replicative experiments (unpaired two-tailed Student's $T$-test).

\section{RESULTS}

\section{Ibal and Kv1.3 channel expression is increased in} frontal cortex of human $A D$ brains

Frontal lobe paraffin-embedded tissue from $10 \mathrm{AD}$ cases and 10 control cases were stained individually for Kv1.3 and microglial marker Iba1 and graded in a blinded manner as described above. There was good agreement between the two raters (intra-class coefficient 0.68 for staining density and 0.73 for staining intensity). In a combined analysis from both raters, cortical regions of $\mathrm{AD}$ brains showed higher intensity $(p=0.002)$ and a trend toward higher density $(p=0.06)$ of Iba1 ${ }^{\text {positive }}$ microglia (Fig. 1). In a similar manner, cortical regions of AD brains had higher Kv1.3 staining intensity $(p=0.03)$ and higher density $(p=0.03)$ of Kv1.3 $3^{\text {positive }}$ cells (Fig. 1). No significant differences in staining intensity or staining density were observed in white matter regions (Supplementary Fig. 2) except for higher Iba1 staining density in AD cases as compared to controls $(p=0.04)$. In $4 \mathrm{AD}$ and 5 control frontal cortical samples, Kv1.3 protein expression as estimated by quantitative immunoblotting was higher in $\mathrm{AD}$ cases as compared to controls in the membraneenriched fractions (Fig. 2). These results suggest that Kv1.3 and Iba1 expression is significantly higher in the cortex of $\mathrm{AD}$ patients as compared to controls.
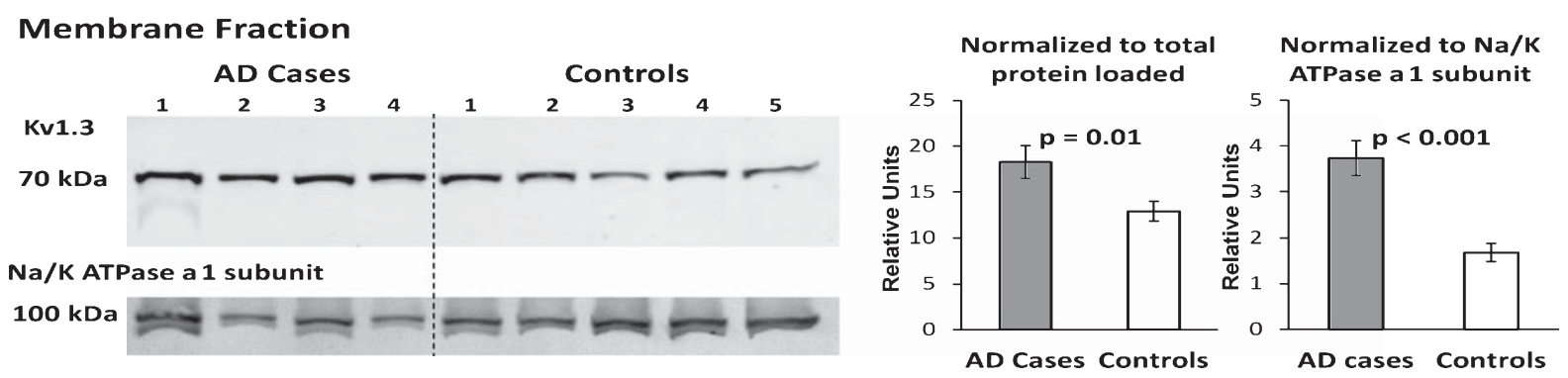

Fig. 2. Kv1.3 expression in frontal cortex of AD and non-AD controls as measured by immunoblotting. Kv1.3 expression was higher in the membrane fraction of $\mathrm{AD}$ brains $(n=4)$ as compared to non-AD controls $(n=5)$. 

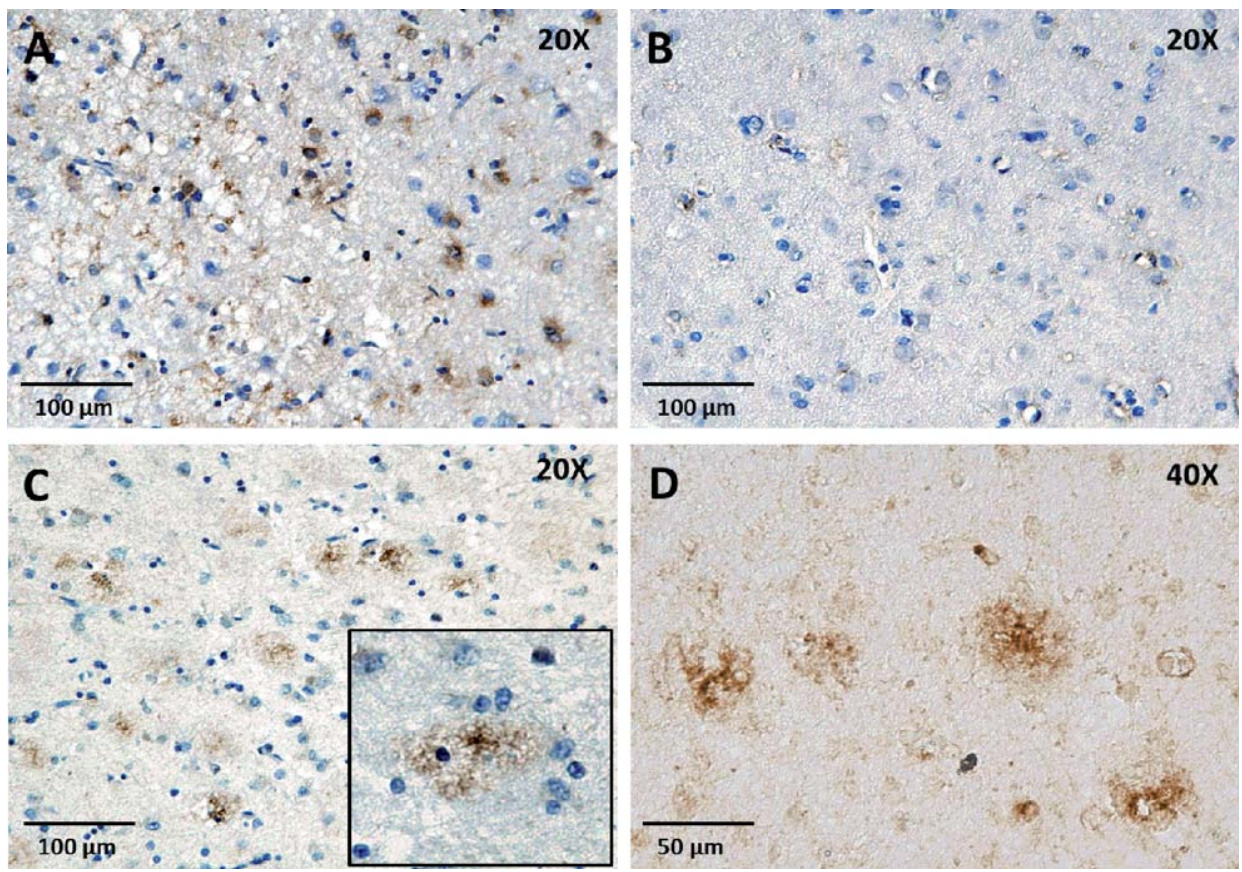

Fig. 3. Patterns of Kv1.3 channel expression in AD brains. Two staining patterns were observed. A) Kv1.3 staining in AD brain: "glial pattern" (20x). B) Secondary antibody control (20x). C) Kv1.3 staining in AD brain: "plaque-like" morphology (10x). (Inset: Higher magnification). D) 40x magnification of a Kv1.3 plaque-like lesion without hematoxylin counterstain.

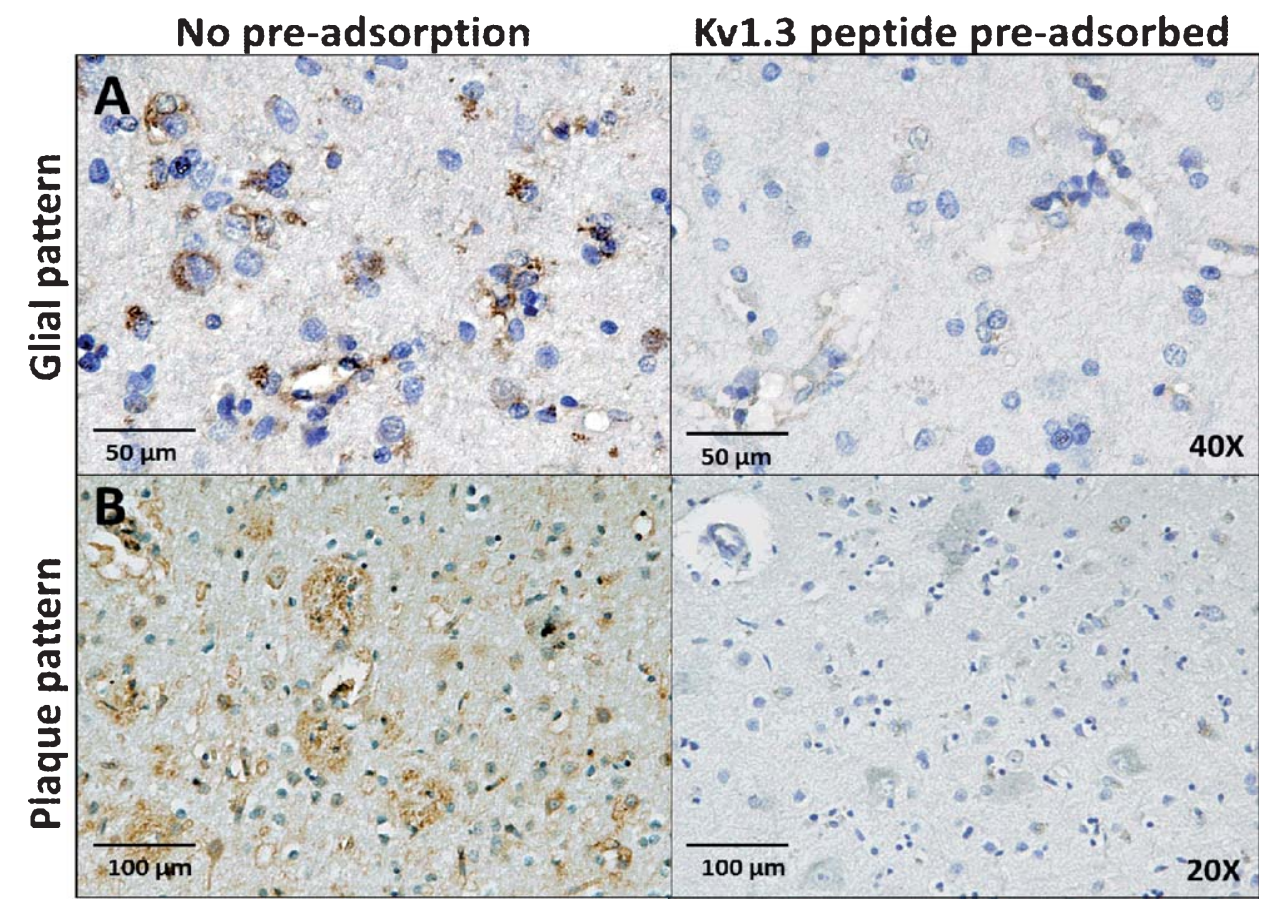

Fig. 4. Specificity of Kv1.3 antibody. Pre-adsorption with Kv1.3 immunizing peptide ( $8 \mu \mathrm{g} / \mathrm{ml})$ abolishes glial (A) as well as plaque-patterns (B) of Kv1.3 staining in frontal cortical regions of AD brain tissue. 


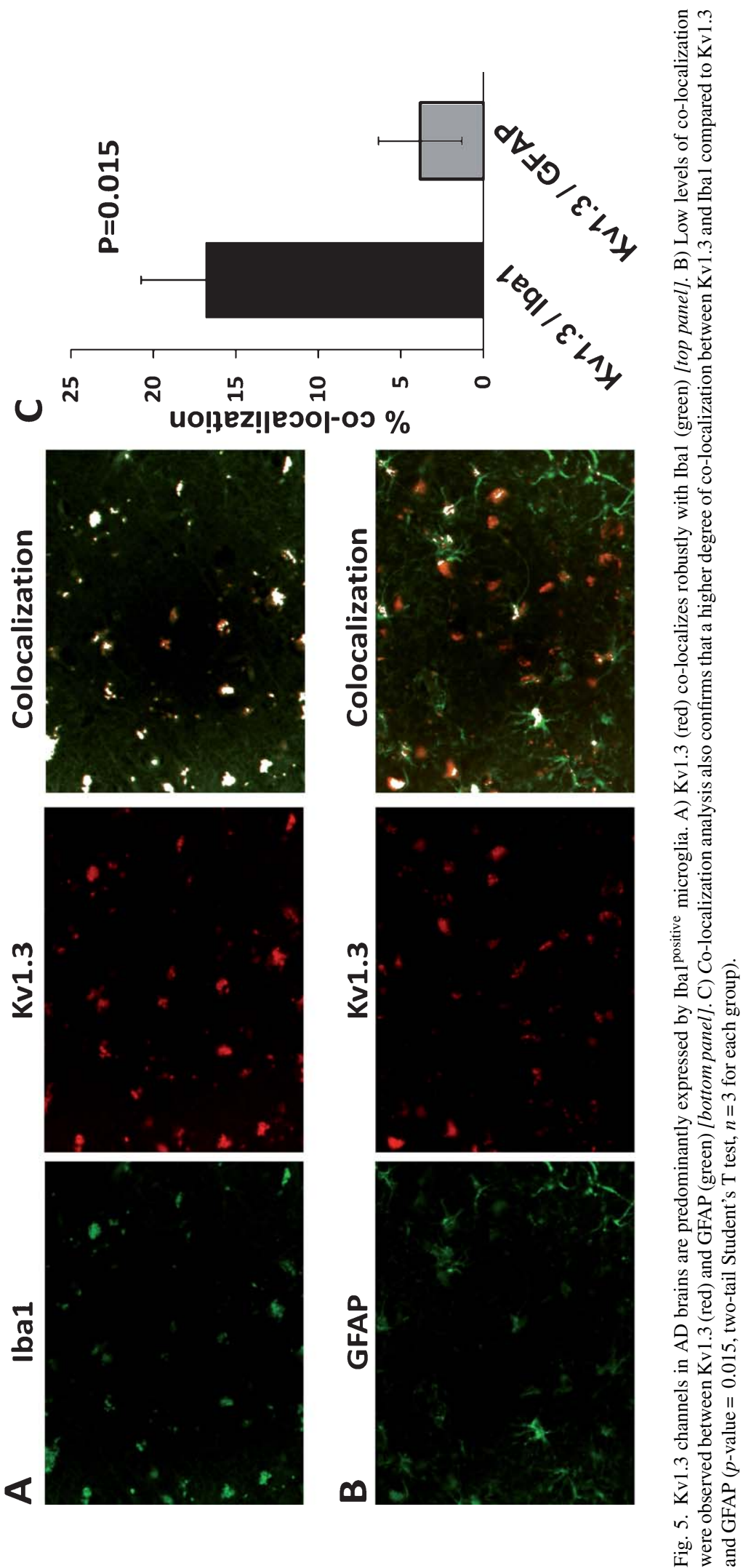



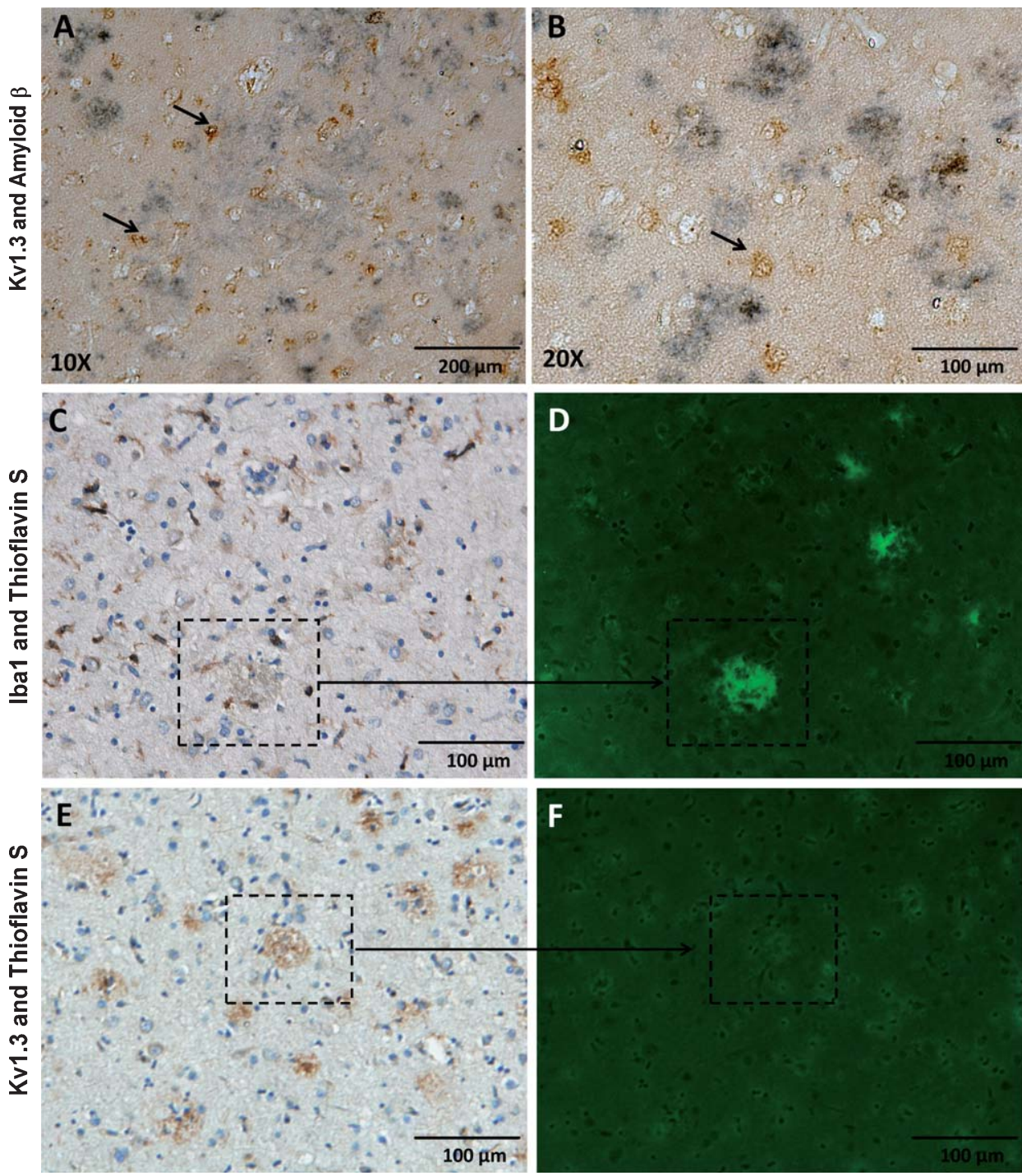

Fig. 6. Kv1.3 $3^{\text {positive }}$ cells are found around $\mathrm{A} \beta$ aggregates in $\mathrm{AD}$ brain. A, B) Kv1.3 ${ }^{\text {positive }}$ cells (brown) aggregate around $\mathrm{A} \beta$ plaques (blueblack). Arrows indicate Kv1.3 $3^{\text {positive }}$ cells (A: 10x, B: 20X). C, D) Iba1 ${ }^{\text {positive }}$ microglia, like Kv1.3 ${ }^{\text {positive }}$ cells, are also found in the vicinity of amyloid plaques (Thioflavin S positive, fluorescent green). E, F) Kv1.3 ${ }^{\text {positive }}$ plaque-like regions are Thioflavin S negative, suggesting that plaque-like regions of Kv1.3 positivity do not represent amyloid plaques.

\section{Patterns of Kv1.3 channel expression in AD brain tissue}

In an un-blinded qualitative analysis of the 10 AD cases, we observed two predominant forms of Kv1.3 staining in the cortex: 1) "Glial-pattern" where $\mathrm{Kv1.3}{ }^{\text {positive }}$ cells appeared to have glial morphology with thin cytoplasmic processes and smaller nuclei (Fig. 3A), morphologically similar to microglia; and 2) "Plaque-like" morphology where Kv1.3 staining was seen in the form of aggregates (Fig. 3C, D). Such a plaque-like pattern could imply Kv1.3 channel aggregation at the site of amyloid plaques or non-specific binding of the antibody to protein aggregates as well as to microglia through FcR binding. Kv1.3 positive plaque-like regions were also Thioflavin- $S^{\text {negative }}$, suggesting that the anti-Kv1.3 antibody was not binding in a non-specific manner to $A \beta$ plaques and also that Kv1.3 plaque-like regions are distinct from $A \beta$ plaques (Fig. 6E, F).

To confirm the specificity of the anti-Kv1.3 antibody used in this study, we pre-adsorbed the anti-Kv1.3 antibody with saturating amounts of the immunogenic peptide before performing IHC. Peptide pre-adsorption abolished both the glial-type (Fig. 4A) and plaque-like patterns (Fig. 4B) of Kv1.3 staining in 
AD brains confirming the specificity of this antibody. Consistent with observations by others [13], the antiKv1.3 antibody was also able to detect Kv1.3 channel expression in the marginal zone in the spleen without significant staining in other regions (data not shown), also confirming the specificity of this antibody for Kv1.3 channels.

\section{Kv1.3 channels are predominantly expressed by microglia in AD brains}

In order to confirm the cellular localization of Kv1.3 in $\mathrm{AD}$ brain tissue, we performed immunofluorescence microscopy using antibodies directed against microglia (Iba1, anti-goat polyclonal IgG, 1:200), astrocytes (GFAP, mouse monoclonal IgG, 1:500), neurons (Tuj1, anti-mouse monoclonal IgG, $1: 100$ ), and Kv1.3 channels (goat anti-rabbit polyclonal, $1: 100)$. Strong co-localization between Kv1.3 and Iba1 and minimal co-localization between Kv1.3 and GFAP was observed (Fig. 4, $p=0.015$ ). Details of this analysis have been illustrated (Supplementary Fig. 1). In $3 \mathrm{AD}$ cortical samples, we also found low levels of Kv1.3 expression in cell bodies but not axons of Tuj1positive neurons (Supplementary Fig. 3) although this analysis was limited by autofluorescence in a significant proportion of cortical neurons (data not shown). These results suggest that Kv1.3 is primarily expressed by microglia in human $\mathrm{AD}$ brain tissue.

\section{Ibal and Kv1.3 channel expression is relation to $A \beta$ deposits}

$\mathrm{A} \beta$ plaques are one of the hallmarks of $\mathrm{AD}$ and activated microglia invade and surround $A \beta$ plaques [14]. We also found aggregation of Iba1 ${ }^{\text {positive }}$ microglia in the vicinity of $A \beta$ plaques identified by Thioflavin-S fluorescent staining (Fig. 6A, B). In dual-chromogen IHC experiments simultaneously probing for Kv1.3 and $A \beta$, we observed numerous Kv1.3 ${ }^{\text {positive }}$ glia in the vicinity of amyloid deposits (Fig. 6C, D; $n=3 \mathrm{AD}$ cases). Kv1.3 expression in our analysis is predominantly limited to microglia. Therefore, the aggregation of Iba1 ${ }^{\text {positive }}$ cells and Kv1.3 expression in the vicinity of $A \beta$ deposits suggests a role for Kv1.3 $3^{\text {positive }}$ microglia in the pathogenesis of $\mathrm{AD}$.

\section{DISCUSSION}

A central role for microglia in AD pathogenesis is supported by results from human genetic studies that confirmed an association between risk of $\mathrm{AD}$ and variations/polymorphisms in genes that encode microglial proteins. A mutation in TREM2, a microglial anti-inflammatory protein that represses microglial cytokine production and neuronal damage [15] confers an increased risk for late-onset AD [4]. Increased expression of microglial protein CD33 is also associated with increased amyloid burden and single nucleotide polymorphisms in CD33 confer an increased risk for $\mathrm{AD}[5,16]$. These results are congruent with data from animal models and support targeting of microglia in $\mathrm{AD}$ as a therapeutic strategy [17]. However, non-selective genetic depletion of microglia does not alter neuropathological features in a mouse model of AD suggesting that anti-microglial strategies should target neurotoxic microglia, while sparing neuroprotective subsets [18]. In addition, an understanding of cellular markers and pathways that define these microglial populations is critical in identifying novel therapeutic targets in $\mathrm{AD}[17,19,20]$. In immune cells such as lymphocytes, potassium channels (Kv1.3, $\mathrm{KCa} 3.1$ ) have been found to regulate cellular functions limited to distinct cell subsets that mediate autoimmunity, a finding of therapeutic relevance in human disease [9, 21]. Potassium channels (Kv1.3, Kv1.5 and $\mathrm{KCa} 3.1)$ and the transient receptor potential cation channel subfamily V member 1 (TRPV1) channel are also expressed by microglia and macrophages [22-25].

Voltage-gated potassium channels are key regulators of excitability in neurons and cardiac muscles; and of membrane potential in non-excitable tissues such as lymphocytes, platelets, macrophages, and microglia. Following membrane depolarization, efflux of potassium ions through open potassium channels in lymphocytes restores the electrophysiological gradient necessary to maintain calcium influx. Down-stream events following calcium entry drive cell migration, proliferation, activation, and cytokine production. Human T cells primarily express $\mathrm{Kv} 1.3$ and $\mathrm{KCa} 3.1$ channels which regulate calcium entry [21]. Upon activation, the majority of $\mathrm{T}$ cells up-regulate $\mathrm{KCa} 3.1$ channels while auto-reactive effector memory $\mathrm{T}$ cells (TEMs) preferentially upregulate Kv1.3 channels, making them susceptible to Kv1.3-specific blockers [26]. Kv1.3 channel-specific peptide toxins (ShK and its analogs [ShK-186, ShK-170, ShK-192] and PAP1) as well as less selective Kv1 channel blockers (BgK and margatoxin) inhibit $\mathrm{T}$ cell proliferation, cytokine release and migration and can effectively ameliorate disease in animal models of autoimmunity (experimental allergic encephalomyelitis, pristane-induced arthritis and delayed-type hypersensitivity) without the side effects of non-specific immunosuppression [26]. 
Microglial potassium channels also demonstrate a pattern of regulation that mirrors the pattern seen in human $\mathrm{T}$ lymphocytes [23, 24]. Resting microglia express Kv1.5 channels, and upon activation by lipopolysaccharide, GM-CSF or interferon- $\gamma$, upregulate Kv1.3 expression [22]. Kv1.3 channels also control microglial proliferation, oxidative burst and neuronal killing [7, 27, 28]. Small conductance calcium-activated potassium channel $\mathrm{KCa} 3.1$ is also expressed in microglia and blockade of this channel abrogates $A \beta$-induced neurotoxicity in murine models of AD [29, 30]. TRPV1 channels seem to be of importance during $A \beta$-induced microglial activation and reactive oxygen species production. Inhibition of TRPV1 by 5-iodo-resiniferatoxin (RTX) abrogates reactive oxygen species production by microglia [25]. However, the "priming" of microglia by A $\beta$ oligomers depends on Kv1.3 channels and is susceptible to inhibition by charybdotoxin and margatoxin but not RTX [8]. Selective regulation of Kv1.3 channel expression by neuroinflammatory cytokines in AD could also alter microglial properties. Transforming growth factor- $\beta$ is a cytokine overexpressed by neurons in $\mathrm{AD}$ and is the only cytokine that is elevated in the cerebrospinal fluid of $\mathrm{AD}$ patients [31] and interestingly, treatment of microglia for $24 \mathrm{~h}$ was found to increase microglial Kv1.3 channel expression by six-fold [32]. The mechanisms underlying microglial polarity (pro-inflammatory M1 or anti-inflammatory or neuroprotective M2) are being defined and it is possible that altered expression of potassium channels could characterize these microglial states $[17,33]$. In vitro data strongly support a role for Kv1.3 channels in $\mathrm{A} \beta$-induced microglial dysfunction [8] but prior to our study, data demonstrating Kv1.3 expression in human AD brain tissue was lacking.

Our results demonstrate that Kv1.3 channels are indeed expressed in cortical microglia at levels higher than non-AD controls as detected by immunohistochemistry. We also found that Kv1.3 expressing microglia are present in proximity to amyloid plaques further strengthening the case for the role of Kv1.3 in AD pathogenesis. No differences in Kv1.3 expression were observed in the white matter of AD cases and controls. These findings suggest that microglial $\mathrm{Kv} 1.3$ upregulation is restricted to cortical gray matter structures only, although we did not specifically look at Kv1.3 expression around white matter $A \beta$ deposits. In addition to increased Kv1.3 expression in cortical microglia in $\mathrm{AD}$ brains, we observed an unexpected "plaque-like" pattern of Kv1.3 expression which was not due to non-specific binding of the Kv1.3 antibody to amyloid plaques. This observation creates the potential for $A \beta$ to interact with Kv1.3 channels. Interestingly, $A \beta_{1-42}$ oligomers, but not soluble $A \beta$, accelerate the activation and inactivation kinetics of $\mathrm{Kv} 1.3$ channels in lipid bilayers without altering channel conductance [34]. Rapid activation and inactivation of Kv1.3 channels could alter calcium fluxes in neurons and microglia but the relevance of this potential Kv1.3$\mathrm{A} \beta$ interaction has not been evaluated. This staining pattern could represent aggregates of microglia, astrocytes, or Kv1.3-expressing neurons and the cellular localization of this Kv1.3 plaque-like pattern needs to be elucidated.

Selective CNS-penetrating Kv1.3 channel blockers could target activated microglia while sparing other glial subtypes that do not express Kv1.3 channels at significant levels. Neurons express a wide array of outward-rectifying potassium channels that predominantly include Kv1.2, 1.4, 1.6, 2.1 and smallconductance potassium (SK) channels, none of which are affected by the highly selective Kv1.3 channel blockers (ShK-186, Shk-170, ShK-192 and PAP-1) $[35,36]$. Kv1.3 channels are expressed in the olfactory bulb and a "super-smeller" phenotype in Kv1.3 -/mice has been observed, but their expression in other cortical brain regions in low $[37,38]$. We, like others, also observed low levels of neuronal Kv1.3 staining in cell bodies of neurons in the frontal cortex in human AD brain tissue [39] although we were unable to clarify whether degenerating neurons in the AD cortex express altered levels of Kv1.3 protein.

An FDA-approved non-specific blocker of Kv channel, 4-aminopyridine, also blocks Kv1.3 channels and has a good safety profile in humans except for seizures at high doses $[40,41]$ that likely result from lowered seizure-threshold due to neuronal potassium channel blockade. In animal studies, treatment with ShK-186 and a lipid soluble Kv1.3 blocker PAP-1 did not increase their susceptibility to influenza and chlamydial infection [26, 42, 43]. A human Phase 1 study of ShK-186 as a novel treatment for autoimmune diseases is also currently underway [44]. Although the neurologic safety profile of selective Kv1.3 blockers in humans has not been demonstrated, the above observations suggest that selective Kv1.3 blockade may not result in significant neurologic or immunosuppressive adverse effects.

The relevance of microglial Kv1.3 channels in neuroinflammation is also likely to extend beyond AD. For example, activated microglia after status epilepticus upregulate a potassium channel electrophysiologically identical to Kv1.3 [45]. HIV-Tat also 
induces Kv1.3 channel expression in microglia and blockade with 4-aminopyridine seems to abrogate neurotoxicity in-vitro [46]. Altered Kv1.3 channel regulation in microglia and blood-derived dendritic cells has also been observed in neuroinflammatory disease states such as radiation-induced brain injury and multiple sclerosis [24, 47].

Our study is limited by the relatively small numbers of cases and controls used. Although the method used for grading of staining was semi-quantitative, inter-observer agreement was acceptable. Our study was restricted to the frontal cortex and whether similar findings are observed in other regions of AD brain will need to be evaluated in future studies. Iba1 was used as a sole marker for migroglia in our experiments. Since blood-derived mononuclear phagocytes recruited to the brain in $\mathrm{AD}$ may also upregulate Iba1, we may have detected Kv1.3 staining on nonmicroglial cells, although we expect these to represent a minority of the Kv1.3 ${ }^{\text {positive }}$ cells in $\mathrm{AD}$ brains. Some Kv1.3 $3^{\text {positive }}$ Iba1 ${ }^{\text {positive }}$ cells were round/oval with poorly visualized processes, both atypical for microglial morphology. This could be explained by the detection of Iba1 ${ }^{\text {positive }}$ non-microglial cells or by $A \beta-$ induced morphological changes observed in microglia in AD brains. Suboptimal staining of microglial processes is also possible as a result of methodological limitations with paraffin-embedded post-mortem brain tissue and by the use of cupric sulfate to reduce autofluorescence by lipofuscin.

In summary, we have demonstrated that Kv1.3 expression is increased in the frontal cortex of humans with AD as compared to non-AD controls. Kv1.3 channels are expressed in the vicinity of $A \beta$ plaques and are primarily expressed by microglia as demonstrated by co-localization between Kv1.3 and Iba1. In context of the growing body of literature suggesting a role for microglia in neurodegeneration, and for potassium channels in microglial physiology, our results provide further evidence that microglial Kv1.3 channels represent therapeutic targets in human neuroinflammatory and neurodegenerative disorders such as AD.

\section{ACKNOWLEDGMENTS}

This work was supported by the Emory Alzheimer's Disease Research Center Grant (P50 AG025688, A.I.L.) and by the American Brain Foundation Clinical Research Training Fellowship in the Neurological Application of Neurotoxins (\#00042351, S.R.). We would also like to thank Prof. George K. Chandy
(Department of Physiology and Biophysics, U.C. Irvine, CA) for his critical reading of the manuscript.

Authors' disclosures available online (http://www.jalz.com/disclosures/view.php?id=2566 ).

\section{SUPPLEMENTARY MATERIAL}

The supplementary material is available in the electronic version of this article: http://dx.doi.org/ 10.3233/JAD-141704.

\section{REFERENCES}

[1] Lehnardt S (2010) Innate immunity and neuroinflammation in the CNS: The role of microglia in Toll-like receptor-mediated neuronal injury. Glia 58, 253-263.

[2] Varnum MM, Ikezu T (2012) The classification of microglial activation phenotypes on neurodegeneration and regeneration in Alzheimer's disease brain. Arch Immunol Ther Exp (Warsz) 60, 251-266.

[3] Hickman SE, Allison EK, El Khoury J (2008) Microglial dysfunction and defective beta-amyloid clearance pathways in aging Alzheimer's disease mice. J Neurosci 28, 8354-8360.

[4] Jonsson T, Stefansson H, Steinberg S, Jonsdottir I, Jonsson PV, Snaedal J, Bjornsson S, Huttenlocher J, Levey AI, Lah JJ, Rujescu D, Hampel H, Giegling I, Andreassen OA, Engedal K, Ulstein I, Djurovic S, Ibrahim-Verbaas C, Hofman A, Ikram MA, van Duijn CM, Thorsteinsdottir U, Kong A, Stefansson K (2013) Variant of TREM2 associated with the risk of Alzheimer's disease. N Engl J Med 368, 107-116.

[5] Bradshaw EM, Chibnik LB, Keenan BT, Ottoboni L, Raj T, Tang A, Rosenkrantz LL, Imboywa S, Lee M, Von Korff A, Morris MC, Evans DA, Johnson K, Sperling RA, Schneider JA, Bennett DA, De Jager PL (2013) CD33 Alzheimer's disease locus: Altered monocyte function and amyloid biology. Nat Neurosci 16, 848-850.

[6] Guerreiro R, Wojtas A, Bras J, Carrasquillo M, Rogaeva E, Majounie E, Cruchaga C, Sassi C, Kauwe JS, Younkin S, Hazrati L, Collinge J, Pocock J, Lashley T, Williams J, Lambert JC, Amouyel P, Goate A, Rademakers R, Morgan K, Powell J, St George-Hyslop P, Singleton A, Hardy J (2013) TREM2 variants in Alzheimer's disease. N Engl J Med 368, 117-127.

[7] Kotecha SA, Schlichter LC (1999) A Kv1.5 to Kv1.3 switch in endogenous hippocampal microglia and a role in proliferation. J Neurosci 19, 10680-10693.

[8] Schilling T, Eder C (2011) Amyloid-beta-induced reactive oxygen species production and priming are differentially regulated by ion channels in microglia. J Cell Physiol 226, 3295-3302.

[9] Rangaraju S, Chi V, Pennington MW, Chandy KG (2009) $\mathrm{Kv} 1.3$ potassium channels as a therapeutic target in multiple sclerosis. Expert Opin Ther Targets 13, 909-924.

[10] Schneider CA, Rasband WS, Eliceiri KW (2012) NIH Image to ImageJ: 25 years of image analysis. Nat Methods 9, 671675.

[11] Guntern R, Bouras C, Hof PR, Vallet PG (1992) An improved thioflavine $\mathrm{S}$ method for staining neurofibrillary tangles and senile plaques in Alzheimer's disease. Experientia 48, 8-10.

[12] Hales CM, Rees H, Seyfried NT, Dammer EB, Duong DM, Gearing M, Montine TJ, Troncoso JC, Thambisetty M, Levey AI, Lah JJ, Wingo TS (2013) Abnormal gephyrin 
immunoreactivity associated with Alzheimer disease pathologic changes. J Neuropathol Exp Neurol 72, 1009-1015.

[13] Wulff H, Knaus HG, Pennington M, Chandy KG (2004) $K+$ channel expression during $\mathrm{B}$ cell differentiation: Implications for immunomodulation and autoimmunity. J Immunol $\mathbf{1 7 3}$, 776-786.

[14] Akiyama H, Barger S, Barnum S, Bradt B, Bauer J, Cole GM, Cooper NR, Eikelenboom P, Emmerling M, Fiebich BL, Finch CE, Frautschy S, Griffin WS, Hampel H, Hull M, Landreth G, Lue L, Mrak R, Mackenzie IR, McGeer PL, O’Banion MK, Pachter J, Pasinetti G, Plata-Salaman C, Rogers J, Rydel R, Shen Y, Streit W, Strohmeyer R, Tooyoma I, Van Muiswinkel FL, Veerhuis R, Walker D, Webster S, Wegrzyniak B, Wenk G, Wyss-Coray T (2000) Inflammation and Alzheimer's disease. Neurobiol Aging 21, 383-421.

[15] Jiang T, Yu JT, Zhu XC, Tan L (2013) TREM2 in Alzheimer's disease. Mol Neurobiol 48, 180-185.

[16] Griciuc A, Serrano-Pozo A, Parrado AR, Lesinski AN, Asselin CN, Mullin K, Hooli B, Choi SH, Hyman BT, Tanzi RE (2013) Alzheimer's disease risk gene CD33 inhibits microglial uptake of amyloid beta. Neuron 78, 631-643.

[17] Saijo K, Glass CK (2011) Microglial cell origin and phenotypes in health and disease. Nat Rev Immunol 11, 775-787.

[18] Colton CA (2009) Heterogeneity of microglial activation in the innate immune response in the brain. J Neuroimmune Pharmacol 4, 399-418.

[19] Grathwohl SA, Kalin RE, Bolmont T, Prokop S, Winkelmann G, Kaeser SA, Odenthal J, Radde R, Eldh T, Gandy S, Aguzzi A, Staufenbiel M, Mathews PM, Wolburg H, Heppner FL, Jucker M (2009) Formation and maintenance of Alzheimer's disease beta-amyloid plaques in the absence of microglia. Nat Neurosci 12, 1361-1363.

[20] Weitz TM, Town T (2012) Microglia in Alzheimer's disease: It's all about context. Int J Alzheimers Dis 2012, 314185.

[21] Cahalan MD, Chandy KG (2009) The functional network of ion channels in T lymphocytes. Immunol Rev 231, 59-87.

[22] Fischer HG, Eder C, Hadding U, Heinemann U (1995) Cytokine-dependent $K+$ channel profile of microglia at immunologically defined functional states. Neuroscience $\mathbf{6 4}$, 183-191.

[23] Kaushal V, Koeberle PD, Wang Y, Schlichter LC (2007) The $\mathrm{Ca} 2+$-activated $\mathrm{K}+$ channel $\mathrm{KCNN} 4 / \mathrm{KCa} 3.1$ contributes to microglia activation and nitric oxide-dependent neurodegeneration. J Neurosci 27, 234-244

[24] Mullen KM, Rozycka M, Rus H, Hu L, Cudrici C, Zafranskaia E, Pennington MW, Johns DC, Judge SI, Calabresi PA (2006) Potassium channels Kv1.3 and Kv1.5 are expressed on blood-derived dendritic cells in the central nervous system. Ann Neurol 60, 118-127.

[25] Schilling T, Eder C (2009) Importance of the non-selective cation channel TRPV1 for microglial reactive oxygen species generation. J Neuroimmunol 216, 118-121.

[26] Beeton C, Wulff H, Standifer NE, Azam P, Mullen KM, Pennington MW, Kolski-Andreaco A, Wei E, Grino A, Counts DR, Wang PH, LeeHealey CJ, S Andrews B, Sankaranarayanan A, Homerick D, Roeck WW, Tehranzadeh J, Stanhope KL, Zimin P, Havel PJ, Griffey S, Knaus HG, Nepom GT, Gutman GA, Calabresi PA, Chandy KG (2006) Kv1.3 channels are a therapeutic target for T cell-mediated autoimmune diseases. Proc Natl Acad Sci U S A 103, 1741417419.

[27] Khanna R, Roy L, Zhu X, Schlichter LC (2001) K+ channels and the microglial respiratory burst. Am J Physiol Cell Physiol 280, C796-806.
[28] Fordyce CB, Jagasia R, Zhu X, Schlichter LC (2005) Microglia Kv1.3 channels contribute to their ability to kill neurons. J Neurosci 25, 7139-7149.

[29] Skaper SD (2011) Ion channels on microglia: Therapeutic targets for neuroprotection. CNS Neurol Disord Drug Targets 10, 44-56.

[30] Maezawa I, Zimin PI, Wulff H, Jin LW (2011) Amyloid-beta protein oligomer at low nanomolar concentrations activates microglia and induces microglial neurotoxicity. J Biol Chem 286, 3693-3706

[31] Swardfager W, Lanctot K, Rothenburg L, Wong A, Cappell J, Herrmann N (2010) A meta-analysis of cytokines in Alzheimer's disease. Biol Psychiatry 68, 930-941.

[32] Schilling T, Quandt FN, Cherny VV, Zhou W, Heinemann U, Decoursey TE, Eder C (2000) Upregulation of Kv1.3 K(+) channels in microglia deactivated by TGF-beta. Am J Physiol Cell Physiol 279, C1123-1134.

[33] Maezawa I, Jenkins PD, Sexton J, Wulff H, Jin LW (2012) The role of potassium channel Kv1.3 in A $\beta$-induced microglia activation. Alzheimers Dement $\mathbf{8}$ (Suppl), P301

[34] Lioudyno MI, Broccio M, Sokolov Y, Rasool S, Wu J, Alkire MT, Liu V, Kozak JA, Dennison PR, Glabe CG, Losche M, Hall JE (2012) Effect of synthetic abeta peptide oligomers and fluorinated solvents on Kv1.3 channel properties and membrane conductance. PLoS One 7, e35090.

[35] Gutman GA, Chandy KG, Adelman JP, Aiyar J, Bayliss DA, Clapham DE, Covarriubias M, Desir GV, Furuichi K, Ganetzky B, Garcia ML, Grissmer S, Jan LY, Karschin A, Kim D, Kuperschmidt S, Kurachi Y, Lazdunski M, Lesage F, Lester HA, McKinnon D, Nichols CG, O'Kelly I, Robbins J, Robertson GA, Rudy B, Sanguinetti M, Seino S, Stuehmer W, Tamkun MM, Vandenberg CA, Wei A, Wulff H, Wymore RS (2003) International Union of Pharmacology. XLI. Compendium of voltage-gated ion channels: Potassium channels. Pharmacol Rev 55, 583-586.

[36] Grissmer S, Nguyen AN, Aiyar J, Hanson DC, Mather RJ, Gutman GA, Karmilowicz MJ, Auperin DD, Chandy KG (1994) Pharmacological characterization of five cloned voltage-gated $K+$ channels, types Kv1.1, 1.2, 1.3, 1.5, and 3.1, stably expressed in mammalian cell lines. Mol Pharmacol 45, 1227-1234.

[37] Veh RW, Lichtinghagen R, Sewing S, Wunder F, Grumbach IM, Pongs O (1995) Immunohistochemical localization of five members of the Kv1 channel subunits: Contrasting subcellular locations and neuron-specific co-localizations in rat brain. Eur J Neurosci 7, 2189-2205.

[38] Fadool DA, Tucker K, Perkins R, Fasciani G, Thompson RN, Parsons AD, Overton JM, Koni PA, Flavell RA, Kaczmarek LK (2004) Kv1.3 channel gene-targeted deletion produces "Super-Smeller Mice" with altered glomeruli, interacting scaffolding proteins, and biophysics. Neuron 41, 389-404.

[39] Rus H, Pardo CA, Hu L, Darrah E, Cudrici C, Niculescu T, Niculescu F, Mullen KM, Allie R, Guo L, Wulff H, Beeton C, Judge SI, Kerr DA, Knaus HG, Chandy KG, Calabresi PA (2005) The voltage-gated potassium channel Kv1.3 is highly expressed on inflammatory infiltrates in multiple sclerosis brain. Proc Natl Acad Sci U S A 102, 11094-11099.

[40] Cornblath DR, Bienen EJ, Blight AR (2012) The safety profile of dalfampridine extended release in multiple sclerosis clinical trials. Clin Ther 34, 1056-1069.

[41] Goodman AD, Brown TR, Edwards KR, Krupp LB, Schapiro RT, Cohen R, Marinucci LN, Blight AR (2010) A phase 3 trial of extended release oral dalfampridine in multiple sclerosis. Ann Neurol 68, 494-502. 
[42] Pennington MW, Beeton C, Galea CA, Smith BJ, Chi V, Monaghan KP, Garcia A, Rangaraju S, Giuffrida A, Plank D, Crossley G, Nugent D, Khaytin I, Lefievre Y, Peshenko I, Dixon C, Chauhan S, Orzel A, Inoue T, Hu X, Moore RV, Norton RS, Chandy KG (2009) Engineering a stable and selective peptide blocker of the Kv1.3 channel in T lymphocytes. Mol Pharmacol 75, 762-773.

[43] Matheu MP, Beeton C, Garcia A, Chi V, Rangaraju S, Safrina O, Monaghan K, Uemura MI, Li D, Pal S, de la Maza LM, Monuki E, Flugel A, Pennington MW, Parker I, Chandy KG, Cahalan MD (2008) Imaging of effector memory T cells during a delayed-type hypersensitivity reaction and suppression by Kv1.3 channel block. Immunity 29, 602-614.
[44] Kineta, http://www.kinetabio.com/pipeline.html, Accessed $01 / 11 / 2014$.

[45] Menteyne A, Levavasseur F, Audinat E, Avignone E (2009) Predominant functional expression of Kv1.3 by activated microglia of the hippocampus after Status epilepticus. PLoS One 4, e6770.

[46] Liu J, Xu P, Collins C, Liu H, Zhang J, Keblesh JP, Xiong H (2013) HIV-1 Tat protein increases microglial outward K(+) current and resultant neurotoxic activity. PLoS One 8, e64904.

[47] Peng Y, Lu K, Li Z, Zhao Y, Wang Y, Hu B, Xu P, Shi X, Zhou B, Pennington M, Chandy KG, Tang Y (2014) Blockade of Kv1.3 channels ameliorates radiation-induced brain injury. Neuro Oncol 16, 528-539. 